\title{
Internal dynamics of the radio-halo cluster A2219: A multi-wavelength analysis ${ }^{\star} \star \star$
}

\author{
W. Boschin ${ }^{1}$, M. Girardi ${ }^{1}$, R. Barrena ${ }^{2,3}$, A. Biviano ${ }^{4}$, L. Feretti $^{5}$, and M. Ramella ${ }^{4}$ \\ 1 Dipartimento di Astronomia, Università degli Studi di Trieste, via Tiepolo 11, 34131 Trieste, Italy \\ e-mail: girardi@ts.astro.it \\ 2 Instituto de Astrofisica de Canarias, 38200 La Laguna, Tenerife, Spain \\ e-mail: rbarrena@ll.iac.es \\ 3 INAF - Telescopio Nazionale Galileo, Roque de Los Muchachos, PO box 565, 38700 Santa Cruz de La Palma, Spain \\ ${ }^{4}$ INAF - Osservatorio Astronomico di Trieste, via Tiepolo 11, 34131 Trieste, Italy \\ e-mail: biviano,ramella@ts.astro.it \\ 5 Istituto di Radioastronomia del C.N.R., via Gobetti 101, 40129 Bologna, Italy \\ e-mail: lferetti@ira.cnr.it
}

Received 19 September 2003 / Accepted 6 November 2003

\begin{abstract}
We present the results of the dynamical analysis of the rich, hot, and X-ray very luminous galaxy cluster A2219, containing a powerful diffuse radio-halo. Our analysis is based on new redshift data for 27 galaxies in the cluster region, measured from spectra obtained at the TNG, with the addition of other 105 galaxies recovered from reduction of CFHT archive data in a cluster region of $\sim 5^{\prime}$ radius $\left(\sim 0.8 h^{-1} \mathrm{Mpc}\right.$ at the cluster distance $)$ centered on the cD galaxy. The investigation of the dynamical status is also performed using X-ray data stored in the Chandra archive. Further, valuable information comes from other bands - optical photometric, infrared, and radio data - which are analyzed and/or discussed, too. We find that A2219 appears as a peak in the velocity space at $z=0.225$, and select 113 cluster members. We compute a high value for the line-ofsight velocity dispersion, $\sigma_{\mathrm{v}}=1438_{-86}^{+109} \mathrm{~km} \mathrm{~s}^{-1}$, consistent with the high average X-ray temperature of $10.3 \mathrm{keV}$. If dynamical equilibrium is assumed, the virial theorem leads to $M \sim 2.8 \times 10^{15} h^{-1} M_{\odot}$ for the global mass within the virial region. However, further investigation based on both optical and X-ray data shows significant signs of a young dynamical status. In fact, we find strong evidence for the elongation of the cluster in the SE-NW direction coupled with a significant velocity gradient, as well as for the presence of substructure both in optical data and X-ray data. Moreover, we point out the presence of several active galaxies. We discuss the results of our multi-wavelength investigation suggesting a complex merging scenario where the main, original structure is subject to an ongoing merger with a few clumps aligned in a filament in the foreground oriented in an oblique direction with respect to the line-of-sight. Our conclusion supports the view of the connection between extended radio emission and merging phenomena in galaxy clusters.
\end{abstract}

Key words. galaxies: clusters: general - galaxies: clusters: individual: Abell 2219 - galaxies: distances and redshifts intergalactic medium - cosmology: observations

\section{Introduction}

In the hierarchical scenario for large-scale-structure formation, mergers are an essential ingredient of galaxy cluster evolution (e.g. White 1997; Evrard \& Gioia 2002).

Send offprint requests to: $\mathrm{W}$. Boschin,

e-mail: boschin@ts.astro.it

* Based on observations made on the island of La Palma with the Italian Telescopio Nazionale Galileo (TNG) operated by the Centro Galileo Galilei of the INAF (Istituto Nazionale di Astrofisica) and with the $1.0 \mathrm{~m}$ Jacobus Kapteyn Telescope (JKT) operated by the Isaac Newton Group at the Spanish Observatorio de Roque de los Muchachos of the Instituto de Astrofisica de Canarias.

$\star \star$ Table 1 is only available in electronic form at the CDS via anonymous ftp to cdsarc.u-strasbg.fr $(130.79 .128 .5)$ or via http://cdsweb.u-strasbg.fr/cgi-bin/qcat?J/A+A/416/839
One of the most recent aspects of the merging phenomenology is the possible connection of cluster mergers with the presence of extended, diffuse radio sources, halos and relics (Feretti 1999; Buote 2002; Giovannini \& Feretti 2002). Radio-halos are located at the cluster center, with typical sizes of $0.5 h^{-1} \mathrm{Mpc}$, regular shape, steep radio spectra and no significant polarization; relics are located in peripheral regions of the clusters, with irregular shape and generally highly polarized spectra (e.g. Feretti \& Giovannini 1996; Giovannini \& Feretti 2002). The synchrotron radio emission of halos and relics demonstrates the existence of large-scale cluster magnetic fields, of the order of $0.1-1 \mu \mathrm{G}$, and of widespread relativistic particles of energy density $10^{-14}-10^{-13} \mathrm{erg} \mathrm{cm}^{-3}$. Although the properties of halos and relics are not well understood, several suggestions for the mechanism transferring energy to the relativistic electrons have been made (e.g. Ensslin 2000). 
Cluster mergers were suggested to provide the large amount of energy necessary for electron re-acceleration and magnetic field amplification (Feretti 1999; Feretti 2002; Sarazin 2002). However, the precise radio-halo formation scenario is still debated (e.g. Ensslin \& Röttgering 2002). Diffuse radio sources are quite uncommon and only recently have we become able to study these phenomena on the basis of sufficient statistics ( $\sim 30$ clusters up to $z \sim 0.3$, e.g. Giovannini et al. 1999; see also Giovannini \& Feretti 2002).

Presently, growing evidence of connection between diffuse emission and cluster merging is based on X-ray data (e.g. Böhringer \& Schuecker 2002; Buote 2002). Studies based on a large number of clusters have found a significant relation between the radio and the X-ray surface brightness (Govoni et al. 2001) and connections between the presence of radiohalos/relics and irregular and bimodal X-ray surface brightness distribution (Schuecker et al. 2001). However, we are far from understanding the whole picture. Unfortunately, to date, optical data is lacking or poorly exploited. Sparse literature concerns some few individual clusters (e.g. Colless \& Dunn 1996; Gómez et al. 2000; Barrena et al. 2002; Mercurio et al. 2003).

Optical data are a powerful way to investigate the presence and the dynamics of cluster mergers (e.g. Girardi \& Biviano 2002), too. The spatial and kinematic analysis of member galaxies allow us to detect and measure the amount of substructure, to identify and analyze possible pre-merging clumps or merger remnants. This optical information is complementary to X-ray information since galaxies and ICM react on different time scales during a merger (see numerical simulations by Roettiger et al. 1997). Moreover, additional information comes from spectral types of member galaxies, since cluster mergers could stimulate star formation in cluster galaxies; thus the spectral signatures of past activity are useful to determine the relevant time-scales (e.g. Bekki 1999; Terlevich et al. 1999).

To investigate the connection between diffuse radio sources and cluster mergers, we have performed spectroscopic observations at TNG and a multi-wavelength analysis of the Abell cluster A2219 (Abell et al. 1989). This cluster shows the presence of a powerful radio-halo (Giovannini et al. 1999; Bacchi et al. 2003) and is a rich, X-ray luminous, hot cluster at moderate redshift $z \sim 0.22-$ Abell richness $=3$ (Abell et al. 1989); $L_{X}(0.1-2.4 \mathrm{keV}) \sim 5 \times 10^{44} h^{-2} \mathrm{erg} \mathrm{s}^{-1}$ (Ebeling et al. 1996); $T_{\mathrm{X}} \sim 10 \mathrm{keV}$ (Rizza et al. 1998). Possible evidence of the young dynamical status of A2219 comes from the ROSAT/HRI X-ray data analysis and strong/weak lensing features (Rizza et al. 1998; Smail et al. 1995; Dahle et al. 2002).

This paper is organized as follows. We present the new redshift data of A2219 in Sect. 2 and the analysis of optical data in Sect. 3. Our analysis of recent X-ray Chandra archival data is shown in Sect. 4. Active galaxies are analyzed and discussed on the basis of multi-wavelength data using mid-IR and radio data in Sect. 5. Finally, in Sect. 6, we summarize and discuss our results presenting a tentative picture of the dynamical status of A2219.

Unless otherwise stated, we give errors at the $68 \%$ confidence level (hereafter c.l.)

Throughout the paper, we assume a flat cosmology with $\Omega_{\mathrm{m}}=0.3$ and $\Omega_{\Lambda}=0.7$. For the sake of simplicity in rescaling we adopt a Hubble constant of $100 \mathrm{~km} \mathrm{~s}^{-1} h^{-1} \mathrm{Mpc}$. In this context, $1^{\prime}$ corresponds to $\sim 0.152 h^{-1} \mathrm{Mpc}$.

\section{Optical observations and data reduction}

Multi-object spectroscopic observations of A2219 were carried out at the TNG telescope in May 2003 (proposal AOT7 ID:18). We used DOLORES/MOS with the LR-B Grism 1, yielding a dispersion of $187 \AA \mathrm{mm}^{-1}$, and the Loral CCD of $2048 \times 2048$ pixels (pixel size of $15 \mu \mathrm{m}$ ). This combination of grating and detector results in dispersions of $2.8 \AA \mathrm{pix}^{-1}$. We have taken 1 MOS mask with 39 slits. We acquired two exposures of $1800.0 \mathrm{~s}$ each. Wavelength calibration was done using arc lamps before or after each exposure (Helium-Argon).

Reduction of spectroscopic data was carried out with IRAF $^{1}$. The signal-to-noise ratio per pixel was computed from the ratio between the signal at $6000 \AA$ and the rms noise within 4000-7000 $\AA$. The resulting average signal-to-noise ratio of our spectra is $S / N \sim 16$.

Radial velocities are determined using the cross-correlation technique (Tonry \& Davis 1979) implemented in the RVSAO package (developed at the Smithsonian Astrophysical Observatory Telescope Data Center). Each spectrum is correlated against six templates for a variety of galaxy spectral types: E, S0, Sa, Sb, Sc, Ir (Kennicutt 1992). The template producing the highest value of $\mathcal{R}$, i.e., the parameter given by RVSAO and related to the signal-to-noise of the correlation peak, is chosen. Also, all spectra and their best correlation functions are examined visually to verify the redshift determination. In some ambiguous cases, generally very late-type galaxies, we use the EMSAO package to confirm the estimated redshift, and in two cases we take the EMSAO redshift as a more reliable estimate of the redshift.

Out of the 39 spectra of objects, 9 turned out to be objects at $z \sim 0$ (included alignment stars for masks), while for 3 cases, with $\mathcal{R} \lesssim 4$, we cannot determine the redshift. Thus, we obtain spectra for 27 galaxies.

We added to our data observations stored in the CFHT archive (proposal ID: 01AF37, P.I.: J.P. Kneib): 1 MOS mask of one $1800 \mathrm{~s}$-exposure with 155 slits. We reduced the spectra with the same procedure adopted for TNG ones. We obtained spectra for 111 galaxies, 6 of which are in common with TNG galaxies. The average $S / N$ of these CFHT spectra is 8 .

To combine our TNG data with those from the CFHT archive, we have to check for a possible zero-point correction. We use the six galaxies with double redshift determination. This allows us to obtain a more rigorous estimate for the redshift errors since the nominal errors as given by the crosscorrelation are known to be smaller than the true errors (e.g. Malamuth et al. 1992; Bardelli et al. 1994; Ellingson \& Yee 1994; Quintana et al. 2000). Thus, for the six galaxies with double redshift determination, we fit the first determination vs. the second one by using a straight line and considering errors in

\footnotetext{
${ }^{1}$ IRAF is distributed by the National Optical Astronomy Observatories, which are operated by the Association of Universities for Research in Astronomy, Inc., under cooperative agreement with the National Science Foundation.
} 
both coordinates (e.g. Press et al. 1992). The fitted line agrees with the one to one relation (slope $=0.996 \pm 0.004$, intercept $=$ $\left.(1 \pm 1) \times 10^{-3}\right)$, but the small value of $\chi^{2}$-probability indicates a poor fit, suggesting that the nominal cross-correlation errors are underestimated. Only when nominal errors are multiplied by $a \sim 2.3$ factor can the observed scatter be explained. Therefore, hereafter we assume that true errors are larger than nominal cross-correlation errors by a factor of 2.3. Slightly smaller correction factors are obtained for nearby clusters (e.g. Malamuth et al. 1992; Bardelli et al. 1994; Quintana et al. 2000).

Our spectroscopic catalogue consists of 132 galaxies sampling a cluster region of $5^{\prime}$ around the dominant galaxy (No. 65), which is a cD galaxy (e.g. Allen et al. 1992).

We also determine the equivalent widths ( $E W$ hereafter) of the emission line [OII] and the absorption line $\mathrm{H} \delta$, which are good indicators of current and recent star formation, respectively. Hereafter all the $E W$ are given in angstroms and are positive if the line is in absorption and negative if the line is in emission. We estimate the minimum measurable $E W$ as the width of a line spanning $2.8 \AA$ (our dispersion) in wavelength, with an intensity three times the rms noise in the adjacent continuum.

A variety of spectroscopic-classification schemes are presented in the literature (see, e.g., Couch et al. 1994; Dressler et al. 1999 and references therein). We use a conservative approach leading to a sparse classification $(45 \%$ of the sample, see Table 1 ). We define $e$-type galaxies as those showing strong active star formation as indicated by the presence of an [OII] line with an equivalent width of $E W([\mathrm{OII}]) \lesssim-15 \AA$ (e.g. Hammer et al. 1997; Postman et al. 1998). Out of galaxies having $S / N>10$ we define as $a$-type those with strong Balmer absorption $E W(\mathrm{H} \delta)>4 \AA$ (e.g. the "post-starburst" galaxies of Couch et al. 1994). We find fourteen "active" galaxies: four of $e$-type - measured $E W([\mathrm{OII}])$ in the range $[-39.3,-14.3] \AA$; ten of $a$-type - measured $E W(\mathrm{H} \delta)$ in the range $[4.0 ; 12.4] \AA$. Out of non-active galaxies with $S / N>10$ those (37) with measured $E W(\mathrm{H} \delta)<3 \AA$ are classified as passive galaxies ( $k$-type) and the remaining nine galaxies, intermediate between $a$ and $k$ are classified as $i$-type. Non-active galaxies do not show significant $[\mathrm{OII}]$ emission and only two $a$-galaxies show a very small [OII] emission (-3.5 ̊).

As far as photometry is concerned, we obtained exposures on a $10^{\prime} \times 10^{\prime}$ unvignetted field centered on $\alpha=16^{\mathrm{h}} 40^{\mathrm{m}} 22^{\mathrm{s}}$, $\delta=46^{\circ} 42^{\prime} 15^{\prime \prime}$ (J2000) through $B$ and $R$ Harris filters using the CCD camera mounted on the $1 \mathrm{~m}$ JKT telescope (at Roque de los Muchachos Observatory, La Palma) in July 2003. We performed several exposures for total integration times of 3000 and $7200 \mathrm{~s}$ in $R$ and $B$ band, respectively, with seeing conditions about $1.4^{\prime \prime}$. We carried out the image reduction (bias, flat and fringing corrections) using the IRAF package and photometry using the AUTOMAG SExtractor procedure (Bertin \& Arnouts 1996). This photometry was calibrated using Landolt's standard fields at the JKT and TNG telescopes and transformed into the Johnson filter system. We estimate that our photometric sample is complete down to $R=19.7$ and $B=21.4$ within the observed field.

We assign $R$ magnitudes to 131 out of the 132 galaxies of our spectroscopic catalogue: the only exception is galaxy
No. 52, which is too close to a very bright star. Out of these 131 galaxies, 129 have assigned magnitudes in $B$ band, too. We measure redshift for galaxies down to $R \sim 20.5 \mathrm{mag}$, but a high level of completeness is obtained only for bright galaxies ( $\sim 66 \%$ completeness for $R<19.7 \mathrm{mag}$ ).

Table 1 lists the velocity catalogue ${ }^{2}$ (see also Fig. 1): identification number of each galaxy, ID (Col. 1); right ascension and declination, $\alpha$ and $\delta$ (J2000, Cols. 2-7); $R$ magnitudes (Col. 8); $B-R$ colours (Col. 9); heliocentric radial velocities, $v=c z_{\odot}$ (in $\mathrm{km} \mathrm{s}^{-1}$, Col. 10) with assumed errors, $\Delta v$, i.e., the nominal ones given by cross-correlation technique multiplied by 2.3 (Col. 11); the code for the spectral classification SC, where "I" and "R" indicate mid-IR and radio emitting galaxies, respectively (see Sect. 5), (Col. 12); the telescope used to obtain the spectra (Col. 13, T and C indicate TNG and CFHT, respectively).

\section{Optical analysis}

\subsection{Member selection and global properties}

According to the analysis of the redshift distribution based on the one-dimensional adaptive kernel technique (Pisani 1993, see also Fadda et al. 1996 and Girardi et al. 1996) A2219 appears as a peak at $z \sim 0.225$ in redshift space (Fig. 2). Out of 132 galaxies with redshift, fourteen are foreground objects and three are background objects. In particular, the foreground galaxies form two peaks, one of six galaxies at $z \sim 0.11$ and one of eight galaxies at $z \sim 0.19$.

To reject possible interloper galaxies in the main peak we apply the procedure of the "shifting gapper" of Fadda et al. (1996), which combines velocity and position information. This procedure rejects galaxies that are too far from the main body of galaxies in rest-frame velocity $\left(v_{\mathrm{rf}}=(v-\bar{v}) /(1+\bar{v} / c)\right)$ within a fixed radial bin shifted along the distance from the cluster center. According to the Fadda et al. prescriptions, we use a gap of $1000 \mathrm{~km} \mathrm{~s}^{-1}$ and a bin of $0.4 h^{-1} \mathrm{Mpc}$, or large enough to include 15 galaxies. For the cluster center, based on the two-dimensional kernel analysis, we find that the densest peak is centered at RA $16^{\mathrm{h}} 40^{\mathrm{m}} 20.56^{\mathrm{s}}$ and Dec $+46^{\circ} 42^{\prime} 42.2^{\prime \prime}$, very close to the $\mathrm{CD}$ galaxy position; thus hereafter we assume the $\mathrm{cD}$ position as the cluster center RA $16^{\mathrm{h}} 40^{\mathrm{m}} 19.87^{\mathrm{s}}$ and Dec $+46^{\circ} 42^{\prime} 41.3^{\prime \prime}$. The shifting gapper procedure rejects two galaxies as non-members. They are indicated by crosses in Fig. 3, which shows the velocity histogram of the 113 selected cluster members. To check the reliability of the member selection procedure, we also apply the method by den Hartog \& Katgert (1996). Of the initial 132 galaxies with redshift we find the same 113 cluster members.

The mean cluster velocity $\bar{v}=67574 \pm 136 \mathrm{~km} \mathrm{~s}^{-1}$ is computed using the biweight estimator (ROSTAT package; Beers et al. 1990). We estimate the line-of-sight velocity dispersion, $\sigma_{v}$, using the biweight estimator and applying the cosmological correction and the usual correction for velocity errors (Danese et al. 1980). We obtain $\sigma_{v}=1438_{-86}^{+109} \mathrm{~km} \mathrm{~s}^{-1}$, where errors are obtained with the bootstrap technique. Assuming that the

\footnotetext{
2 The coordinates of non-galaxy objects are available upon request.
} 


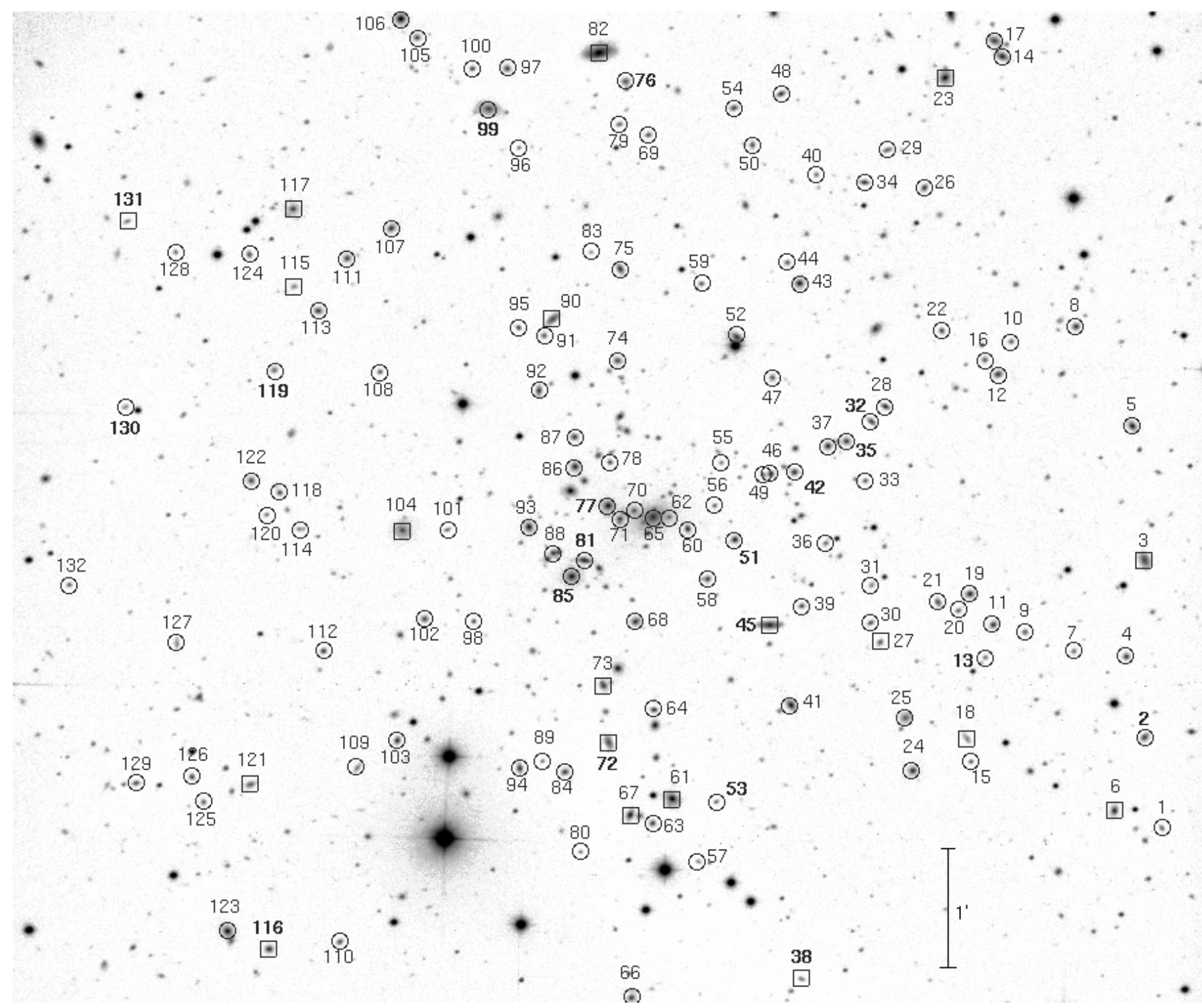

Fig. 1. $R$-band image of A2219 (North at top and East to left). Galaxies with successful velocity measurements are labeled as in Table 1. Circles and boxes indicate cluster member and non-member galaxies, respectively. Labels in bold indicate active galaxies (see text).

system is in dynamical equilibrium, the value of $\sigma_{v}$ leads to a value of the radius of the collapsed, quasi-virialized region of $R_{\mathrm{vir}} \sim R_{200}=\sqrt{3} \sigma_{v} /[10 \mathrm{H}(z)] \sim 2.2 h^{-1} \mathrm{Mpc}$ (Carlberg et al. 1997) and a virial mass estimate of $M\left(<R_{\text {vir }}\right)=2.8_{-0.7}^{+0.8} \times$ $10^{15} h^{-1} M_{\odot}$ (Girardi et al. 1998; Girardi \& Mezzetti 2001).

The high value of $\sigma_{v}$ is confirmed if we apply a tighter member selection (i.e., using a bin of $0.25 h^{-1} \mathrm{Mpc}$ in the shifting gapper): only another three galaxies are rejected -those with the lowest velocities- resulting in $\sigma_{v}=1355_{-82}^{+82} \mathrm{~km} \mathrm{~s}^{-1}$ for the remaining galaxies. Moreover, we compute the differential and integral mean velocity and velocity dispersion profiles in Fig. 4: this analysis shows that the global values of $\bar{v}$ and $\sigma_{v}$ are already reached within the central cluster region of $0.1-0.2 h^{-1} \mathrm{Mpc}$. The conclusion of the above analyses is that contamination by obvious field interlopers and/or close cluster companions cannot explain the high value of the global velocity dispersion. More probably, this value is connected to the peculiarity of the internal dynamics of the cluster itself, which will be analyzed below.

\subsection{Internal dynamics}

As a first step in the study of internal dynamics we analyze the velocity distribution. We consider three estimators of Gaussianity: the kurtosis; the skewness, and the scaled tail index (see, e.g., Beers et al. 1991). We find no significant evidence that the velocity distribution differs from a Gaussian. Moreover, we do not find any evidence of a peculiar velocity of the $\mathrm{cD}$ galaxy with respect to the average velocity of cluster members (see Gebhardt \& Beers 1991).

To investigate the velocity field we divide galaxies in low and high-velocity samples using the median cluster velocity and check the difference between the two distributions of galaxy positions. Figure 5 shows that low and high-velocity galaxies are segregated roughly along the $E-W$ direction. The two distributions are different at the $99.6 \%$ c.l. according to the two-dimensional Kolmogorov-Smirnov test (hereafter 2DKStest; see Fasano \& Franceschini 1987, as implemented by Press et al. 1992). To estimate the direction of the velocity gradient 


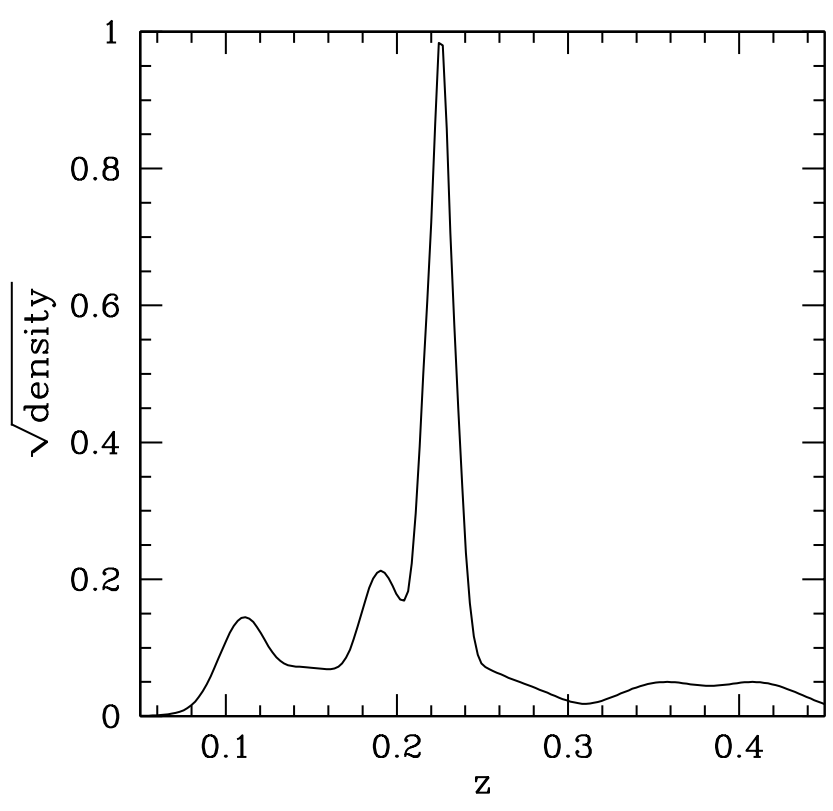

Fig. 2. Square root of the redshift galaxy density, as provided by the adaptive-kernel reconstruction method. Units on the $y$ axis are arbitrary.

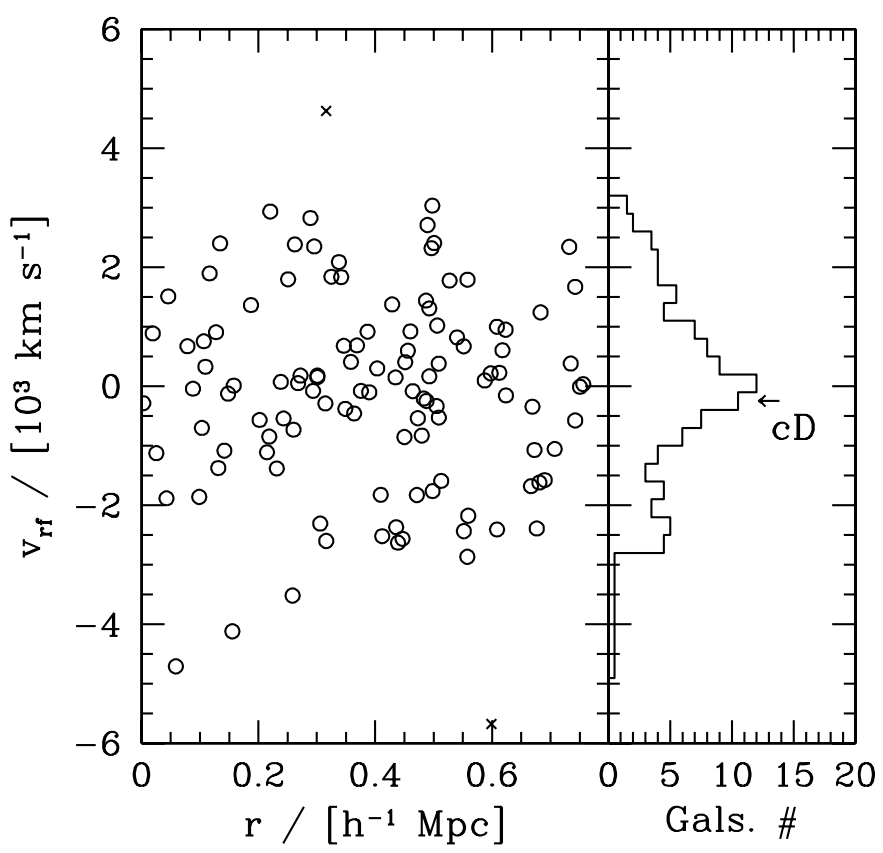

Fig. 3. Left panel: rest-frame velocities $v_{\mathrm{rf}}$ vs. projected cluster-centric distance $r$ of galaxies in the main peak of Fig. 2. Circles indicate cluster members; crosses indicate the two galaxies rejected by the "shifting gapper" procedure. Right panel: histogram of rest-frame velocities of selected 113 cluster members. The arrow indicates the $\mathrm{cD}$ galaxy velocity.

we perform a multiple linear regression fit to the observed velocities with respect to the galaxy positions in the plane of the sky (see also den Hartog \& Katgert 1996; Girardi et al. 1996): we find a PA $=79_{-20}^{+22}$ degrees (measured counter-clock-wise from the North, see Fig. 5).

Moreover, we compute the mean velocity and LOS velocity dispersion separately for each of the four samples

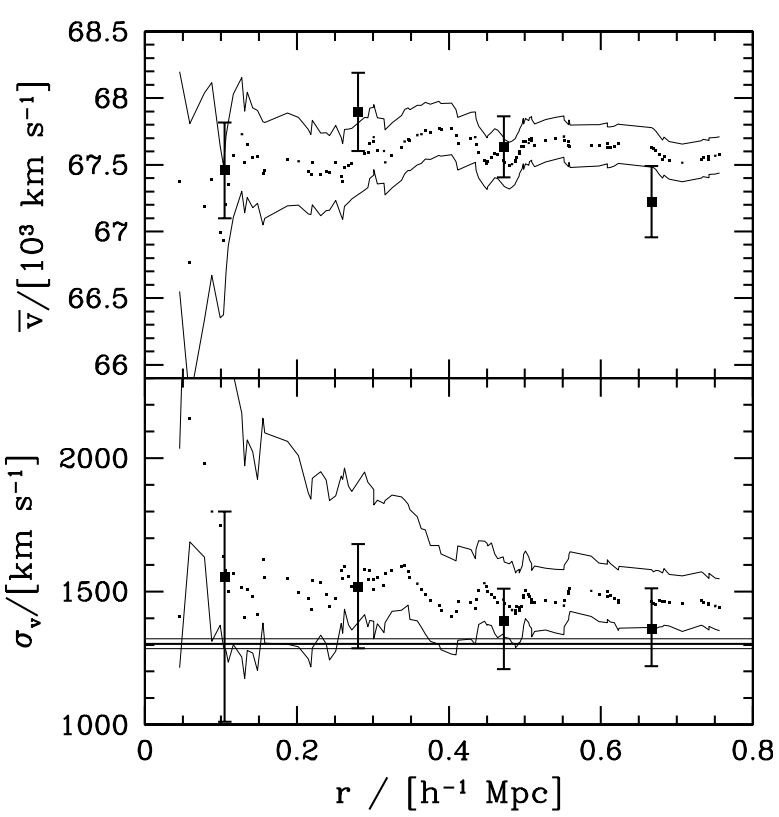

Fig. 4. Differential (big squares) and integral (small points) mean velocity and line-of-sight velocity dispersion profiles. For the differential profiles, the results for four annuli from the cluster center, each of $0.2 \mathrm{~h}^{-1} \mathrm{Mpc}$, are shown. For the integral profiles, the mean and dispersion at a given (projected) radius from the cluster center is estimated by considering all galaxies within that radius. The error bands at the $68 \%$ c.l. are shown. In the lower panel, the horizontal lines represent X-ray temperature with the respective errors transformed in $\sigma_{v}$ (assuming $\beta_{\text {spec }}=1$ where $\beta_{\text {spec }}=\sigma_{v}^{2} /\left(k T_{\mathrm{X}} / \mu m_{\mathrm{p}}\right)$, with $\mu$ the mean molecular weight, $m_{\mathrm{p}}$ the proton mass, and $T_{\mathrm{X}}$ the X-ray temperature).

Table 2. Mean galaxy velocities and velocity dispersion (in $\mathrm{km} \mathrm{s}^{-1}$ ) in four cluster sectors (see text). $N$ indicates the number of member galaxies in each sector.

\begin{tabular}{lccc}
\hline \hline Sector & $N$ & $\bar{v}$ & $\sigma_{v}$ \\
\hline NE & 30 & $66712 \pm 272$ & $1458_{-172}^{+305}$ \\
SE & 26 & $67213 \pm 330$ & $1642_{-152}^{+271}$ \\
SW & 25 & $68007 \pm 288$ & $1403_{-22}^{+248}$ \\
NW & 31 & $68128 \pm 187$ & $1018_{-109}^{+161}$ \\
\hline
\end{tabular}

corresponding to the NE, SE, SW and NW cluster sectors. Table 2 shows that mean velocities are larger in the Western than in the Eastern sectors in agreement with the above analyses. For $\sigma_{v}$, this is largest in the SE sector and smallest in the NW sector: the difference is significant at the $98.7 \%$ c.l. according to the F-test (Press et al. 1992).

To check for the presence of three-dimensional substructure we compute the $\Delta$-statistics devised by Dressler \& Schectman (1988) and establish its significance running 1000 Monte Carlo simulations, in which we randomly shuffle galaxy velocities. The signal of substructure is significant at the 95\% c.1. In Fig. 6 we plot the distribution on the sky of all galaxies, each marked by a circle: the larger the circle, the larger is the deviation $\delta_{\mathrm{i}}$ of the local parameters from the global cluster parameters, i.e., there is more evidence for substructure. The most likely 


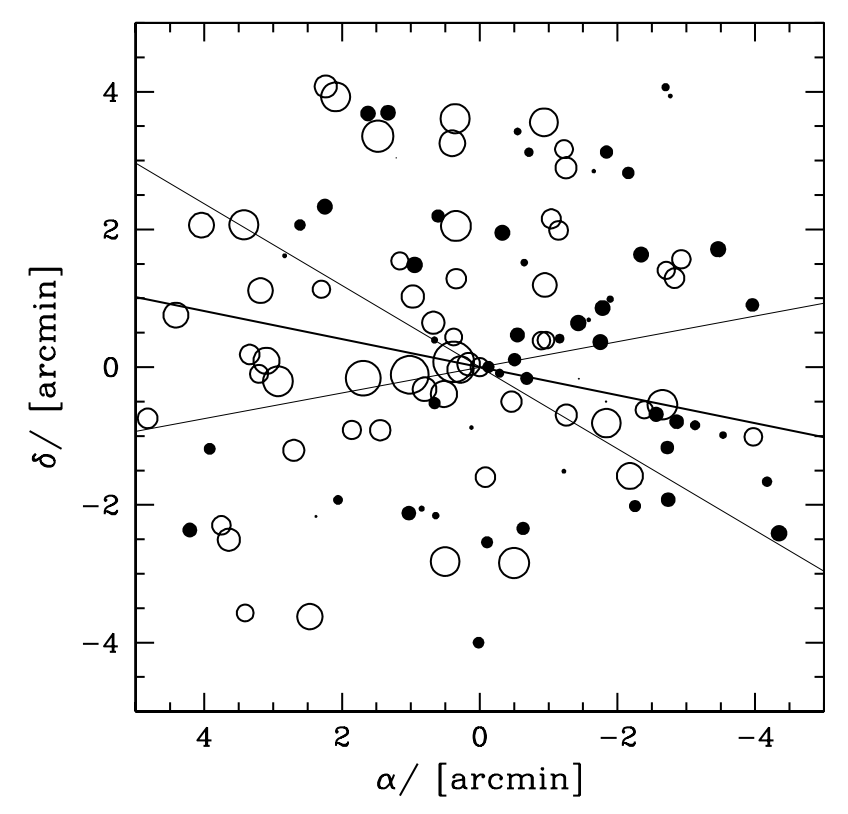

Fig. 5. Spatial distribution on the sky of 113 cluster members. Open and solid circles indicate low-and high-velocity galaxies: the larger the symbol, the smaller is the radial velocity. The plot is centered on the cluster center (coincident with the $\mathrm{cD}$ galaxy). The solid and faint lines indicate the position angle of the cluster velocity gradient and relative errors, respectively.

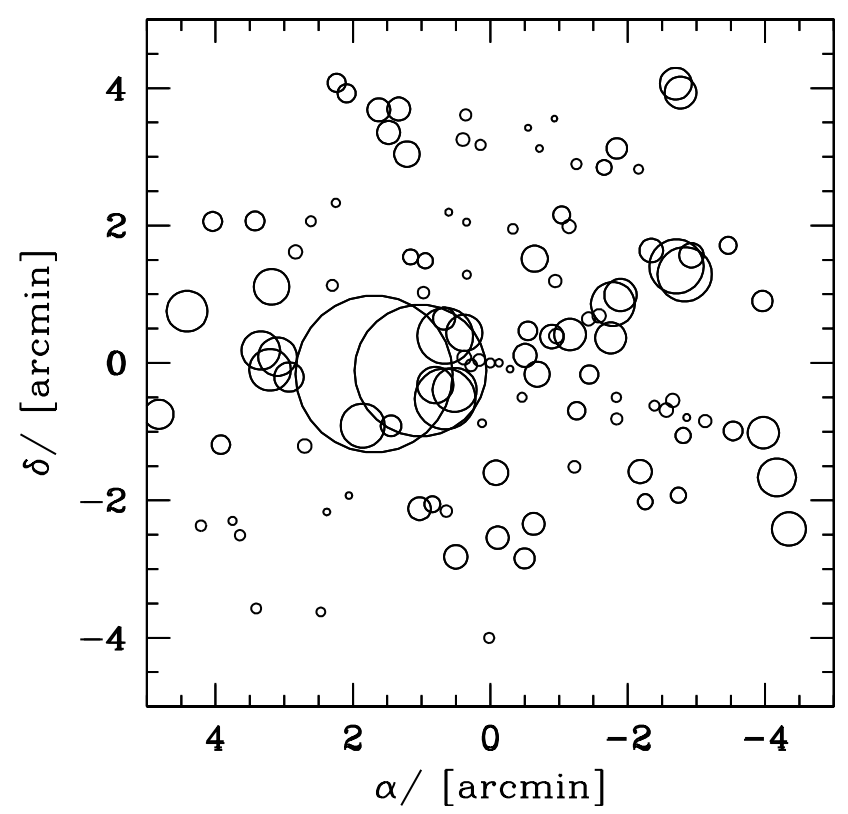

Fig. 6. Spatial distribution on the sky of the 113 cluster members, each marked by a circle: the larger the circle, there is more evidence for substructure (according to the Dressler \& Schectman test, see text). The plot is centered on the cluster center.

substructure lies close to the cluster center roughly to the SE of the cD galaxy.

\subsection{Colour segregation and 2-D galaxy distribution}

We check for possible colour segregation of galaxies using the 110 member galaxies for which we have $B-R$ colours.

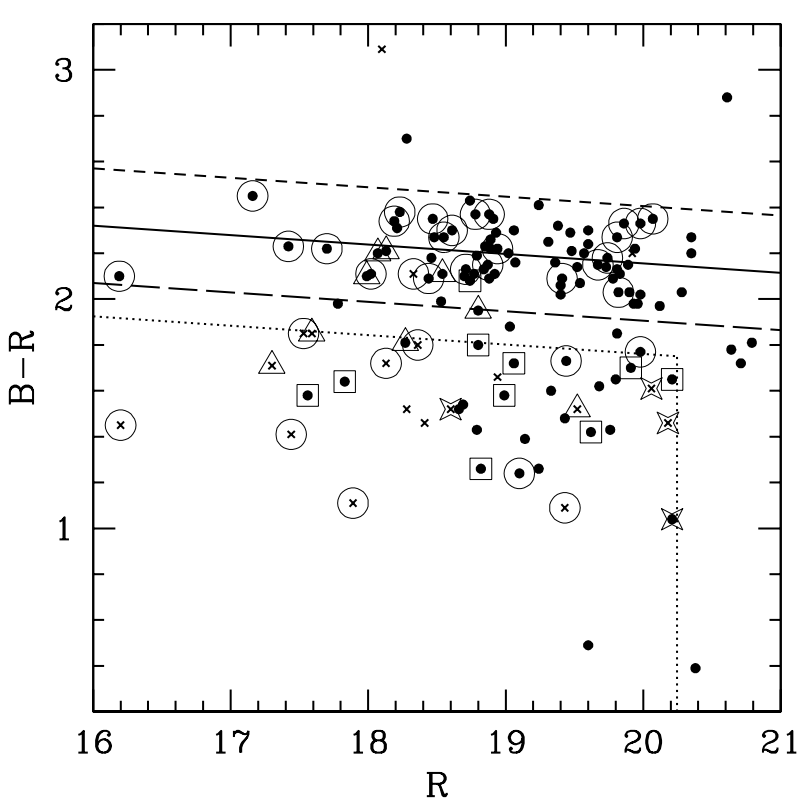

Fig. 7. $B-R$ vs. $R$ diagram for galaxies with available spectroscopy: small dots and crosses denote cluster and field members, respectively. Large symbols denotes spectroscopic classified galaxies: circles, triangles, squares, and stars indicate $k-, i-, a_{-}$, and $e$-galaxies, respectively. The solid line gives the best-fit CMR; the dashed lines are drawn at $\pm 0.25 \mathrm{mag}$ from the CMR. According to our working definition in Sect. 3.3 cluster members are divided in: blue and red galaxies (below and above the long-dashed line, respectively); very red galaxies lie above the solid line. The left-down region delimited by the dotted lines defines the locus of Butcher-Oemler galaxies (Sect. 5).

We obtain a correlation between $\left|v_{\mathrm{rf}}\right|$ and colour (at the $96.3 \%$ c.1.). We divide the sample in two subsamples with colours bluer and redder than the median $B-R$ colour $=2.12$. We obtain that the velocity dispersions of the two subsamples differ at the $99.5 \%$ c.l. $\left(\sigma_{v}=1627_{-96}^{+144}\right.$ and $\sigma_{v}=$ $1100_{-160}^{+190} \mathrm{~km} \mathrm{~s}^{-1}$ for the $B-R<2.12$ and $B-R>2.12$ galaxies, respectively).

To further investigate the above difference we also use the colour-magnitude relation (hereafter CMR), which indicates the early-type galaxy locus,

$B-R=2.976-0.041 \cdot R$,

obtained using a 2 sigma-clipping fitting procedure (see Fig. 7). $B l u e$ objects are defined to be those galaxies at least $0.25 \mathrm{mag}$ bluer in $B-R$ than the colour of the CMR. These blue galaxies have typically $B-R<2$, thus include Sa spiral galaxies and exclude elliptical galaxies (according to the expected typical colours). The remaining objects, which we define the red sample, should consist mostly of ellipticals and lenticulars. We further divide red galaxies in two subsamples: very red and notvery red galaxies, depending on their location above or below the CMR. Table 3 shows $\bar{v}$ and $\sigma_{v}$ estimates for blue and red galaxies, and separately for very and not-very red galaxies. The populations of very red and not-very red galaxies are different. The $\sigma_{v}$ of not-very red galaxies is larger than the $\sigma_{v}$ of very red ones (at the $99.5 \%$ c.l. according to the F-test). Moreover, the mean velocity of not-very red galaxies is smaller than that of very red ones (at the $99.5 \%$ c.l. according to the means-test). 
Table 3. Mean velocities and velocity dispersions (in $\mathrm{km} \mathrm{s}^{-1}$ ) for galaxies with different colors (see text). $N$ is the number of galaxies of each type.

\begin{tabular}{lccc}
\hline \hline \multicolumn{1}{c}{ Population } & $N$ & $\bar{v}$ & $\sigma_{v}$ \\
\hline blue galaxies & 33 & $67676 \pm 255$ & $1439_{-139}^{+177}$ \\
red galaxies & 77 & $67568 \pm 163$ & $1423_{-123}^{+146}$ \\
(not very) red galaxies & 40 & $66942 \pm 265$ & $1655_{-168}^{+15}$ \\
(very) red galaxies & 37 & $67969 \pm 173$ & $1032_{-126}^{+172}$ \\
\hline
\end{tabular}

We also analyze the two-dimensional spatial distribution of galaxies. First, we consider bright cluster members, i.e. the sample of 80 galaxies with $R<19.7$ for which we have a good level of spatial completeness (see Sect. 2). Figure 8 shows the presence of two clumps in the cluster center which defines a SE-NW elongated structure $\left(\mathrm{PA} \sim 110^{\circ}\right)$ : the main peak roughly coincides with the $\mathrm{cD}$ position, and the secondary peak - at the West - traces the filament of high velocity galaxies well visible in Fig. 1 (from No. 49 to No. 28). The $\mathrm{cD}$ also shows an elongation in the SE-NW direction described by the position angle on the celestial sphere PA $=114 \pm 3$ degrees (Smail et al. 1995).

Then, to study fainter galaxies and to work with much more robust statistics, we consider the $B$ and $I$ photometric data of Smail et al. (1998) and define likely members using the CMR. Specifically, we select 484 likely cluster members considering objects $(I<22$, stellar index $<0.9)$ within $0.25 \mathrm{mag}$ from the $B-I=5.398-0.115 \cdot I$. The top panel of Fig. 9 shows a main structure centered on the $\mathrm{cD}$ galaxy and elongated in the SE-NW direction, with a PA of $\sim 110^{\circ}$ for the central region (along the filament described above) and a larger PA for the external region. A similar structure is also traced by the subsample of very red galaxies - those above the CMR (Fig. 9 middle panel). Instead, the distribution of not very red galaxies shows three main clumps aligned in the NE-SW direction (Fig. 9 bottom panel). In particular, the densest peak is located at about $1^{\prime}$ South-East of the $\mathrm{cD}$, in the position of the substructure suggested by the Dressler-Schectman test (Sect. 3.2).

\section{X-ray observation and analysis}

The X-ray analysis of A2219 is performed on the archival data of the Chandra ACIS-S observation 800072 (exposure ID \#896, P.I.: J. Houck). The pointing has an exposure time of $42.8 \mathrm{ks}$. Data reduction is performed using the package $\mathrm{CIAO}^{3}$ (Chandra Interactive Analysis of Observations) on chip S3 (field of view $\sim 8.5^{\prime} \times 8.5^{\prime}$ ). First, we remove events from the level 2 event list with a status not equal to zero and with grades one, five and seven. Then, we select all events with energy between 0.3 and $10 \mathrm{keV}$. In addition, we clean bad offsets and examine the data, filtering out bad columns and removing times when the count rate exceeds three standard deviations from the mean count rate per $3.3 \mathrm{~s}$ interval. We then clean S3 chip for flickering pixels, i.e., times where a pixel has events in two

\footnotetext{
${ }^{3}$ CIAO is freely available at http://asc.harvard.edu/ciao/
}

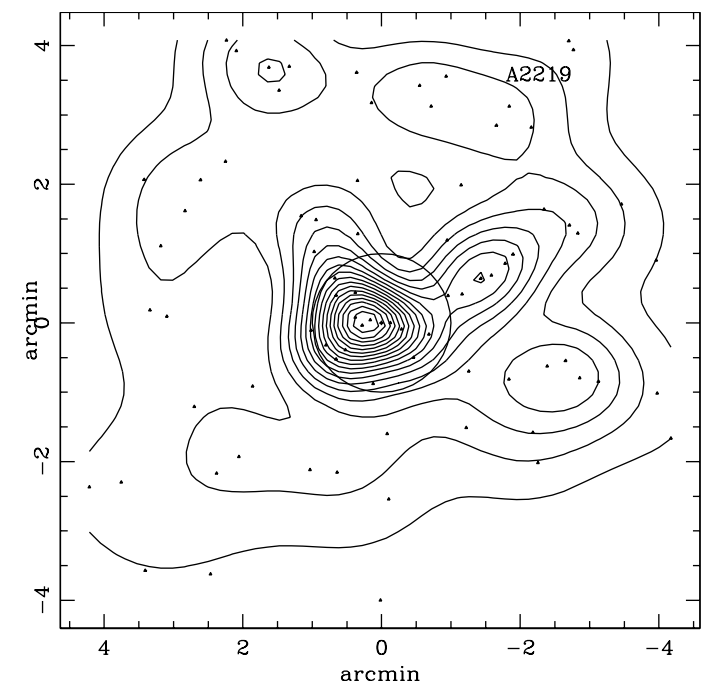

Fig. 8. Spatial distribution on the sky and relative isodensity contour map of 80 bright cluster $(R<19.7$ galaxies), obtained with the adaptive-kernel method (cf. Pisani 1993, 1996). The plots are centered on the cluster center. The $1^{\prime}$ circle is centered on the $\mathrm{cD}$ galaxy.

sequential $3.3 \mathrm{~s}$ intervals. The resulting exposure time for the reduced data is $42.3 \mathrm{ks}$.

In Fig. 10 we plot an $R$-band image of the cluster with superimposed the X-ray contour levels of the Chandra image and the radio contour levels of a low-resolution VLA image by Bacchi et al. (2003). The elongated shape of the cluster is clearly visible. Using the CIAO package Sherpa we fit an elliptical Beta model to the X-ray photon distribution to quantify the departure from the spherical shape. The model is defined as:

$f(x, y)=f(r)=A /\left[1+\left(r / r_{0}\right)^{2}\right]^{\alpha}$

where the radial coordinate $r$ is defined as $r(x, y)=\left[X^{2}(1-\right.$ $\left.\epsilon)^{2}+Y^{2}\right]^{1 / 2} /(1-\epsilon), X=\left(x-x_{0}\right) \cos \theta+\left(y-y_{0}\right) \sin \theta$ and $Y=\left(y-y_{0}\right) \cos \theta-\left(x-x_{0}\right) \sin \theta$. Here $x$ and $y$ are physical pixel coordinates on chip S3. The best fit centroid position is located 12" South-East of the dominant galaxy. The best fit core radius, the ellipticity and the position angle are $r_{0}=69.7 \pm 1.3 \operatorname{arcsec}\left(177 \pm 3 h^{-1} \mathrm{kpc}\right), \epsilon=0.339 \pm 0.006$ and $\mathrm{PA}=128.8 \pm 0.6$ degrees, respectively. The elongated shape of the cluster is also in agreement with Rizza et al.'s (1998) result, which showed evidence of elongations and excesses in the X-ray emission relative to a circular model on ROSAT data (see their Table 7).

To detect possible substructures in A2219 we perform a wavelet analysis by running the task CIAO/Wavdetect on the chip S3. The task was run on different scales to search for substructures with different sizes. The significance threshold $^{4}$ was set at $10^{-6}$. The results are shown in Fig. 10. Thick ellipses represent four significant substructures found by Wavdetect. The principal one, located at RA $16^{\mathrm{h}} 40^{\mathrm{m}} 20.1^{\mathrm{s}}$ and Dec $+46^{\circ} 42^{\prime} 45^{\prime \prime}$, is centered on the $\mathrm{cD}$ galaxy. There are two more significant peaks in the core of the cluster

\footnotetext{
4 see Sect. 11.2 of the CIAO Detect Manual (software release version 2.2.1, available at the WWW site http://cxc.harvard.edu/ ciao/manuals.html).
} 

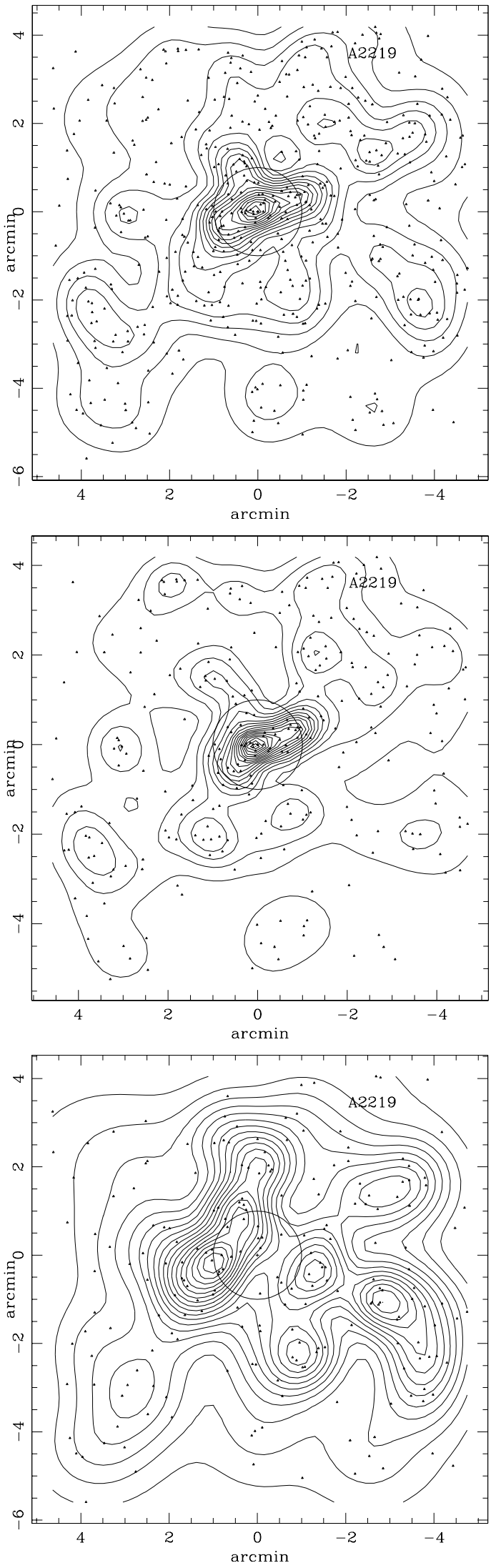

Fig. 9. Same as Fig. 8 but for likely cluster members selected on the basis of the $B-I$ CMR in the photometric sample of Smail et al. (1998). Top panel: the $484 I<22$ galaxies. Middle and bottom panels: two subsamples corresponding to very red and not very red galaxies (273 and 211 galaxies, respectively). located at RA $16^{\mathrm{h}} 40^{\mathrm{m}} 23.1^{\mathrm{s}}$ and Dec $+46^{\circ} 42^{\prime} 19^{\prime \prime}$, and at RA $16^{\mathrm{h}} 40^{\mathrm{m}} 21.6^{\mathrm{s}}$ and Dec $+46^{\circ} 42^{\prime} 26^{\prime \prime}$, respectively. Finally, outside the core, we find a fourth structure at RA $16^{\mathrm{h}} 40^{\mathrm{m}} 27.7^{\mathrm{s}}$ and Dec $+46^{\circ} 41^{\prime} 13^{\prime \prime}$, about $2^{\prime}$ South-East of the dominant galaxy. The presence of a photon excess in this position is also evident from Fig. 11, where we plot the residual map of the elliptical Beta model fit. A peak of positive residuals is observed at the position of the fourth structure.

For the spectral analysis of the cluster X-ray photons, we first compute a global estimate of the ICM temperature. The temperature is computed from the spectrum of the cluster within a circular aperture of $3^{\prime}$ radius around the cluster center. Fixing the absorbing galactic hydrogen column density at $1.75 \times 10^{20} \mathrm{~cm}^{-2}$, computed from the HI maps by Dickey \& Lockman (1990), we fit a Raymond-Smith (1977) spectrum using the CIAO package Sherpa with a $\chi^{2}$ statistics. We find a best fitting temperature of $T_{\mathrm{X}}=10.3 \pm 0.3 \mathrm{keV}$, in agreement with previous estimates by Allen (2000) and White (2000).

To detect the possible presence of temperature gradients in the cluster we divide the cluster in eight sectors, as shown in Fig. 12, and compute the temperature in each of them. We find a temperature gradient from the cooler SE sectors to the hotter NW ones. This pattern is also confirmed by a softness ratio analysis of the Chandra image. We define the softness ratio as $S R \equiv(S-H) /(S+H)$, where $S$ is the number count of photons with soft energies in the range $0.5-2 \mathrm{keV}$, while $H$ is the number count of photons with hard energies in the range $2-7 \mathrm{keV}$. Then we count soft and hard photons in circles with 50 pixel aperture radius sliding on a $200 \times 200$ pixel grid. Background photon counts are subtracted using an ACIS background event file by Markevitch. The background-subtracted values of the softness ratios are plotted in Fig. 13. In this figure different grey levels identify regions with different $S R$ s. High $S R$ (lower temperature) regions are concentrated mainly in sectors $1,2,3$ and 4, in agreement with the temperature map shown in Fig. 12.

\section{Active galaxies}

The star formation properties of the galaxies in our sample are examined on the basis of the spectral classification introduced in Sect. 2 (see Table 1). Out of 60 classified galaxies, 46 are member galaxies: 29/37 $k$-galaxies, 6/9 $i$-galaxies, $1 / 4$ $e$-galaxy, and 10/10 $a$-galaxies. The relative fraction of cluster $a$-galaxies is $22 \%$. For different $E W(\mathrm{H} \delta)$ levels we find $13 \%$ and $37 \%$ ( $>5$ and $>3 \AA$, respectively). For comparison, Poggianti et al. (1999) found $21 \%$ of $k+a / a+k$ galaxies with $E W(\mathrm{H} \delta)>3 \AA$ and Balogh et al. (1999) found a fraction $a \lesssim 4 \%$ of $K+A$ with $E W(\mathrm{H} \delta)>5 \AA$. An open question is whether strong $\mathrm{H} \delta$ galaxies are typical of cluster environments (cf., e.g., Poggianti et al. 1999 with Balogh et al. 1999) since these galaxies could be produced by a cluster merger (Bekki 1999). Very interestingly, we find 0/14 $a$-galaxies in the field compared to $10 / 46$ in cluster environment.

We check for possible spectral segregations of cluster galaxies both in position and in velocity space by comparing the dynamics of passive $k$-galaxies with those of active $e$-plus $a$-galaxies. We find a larger velocity dispersion in the case of active galaxies, but the difference is not significant. Moreover, 


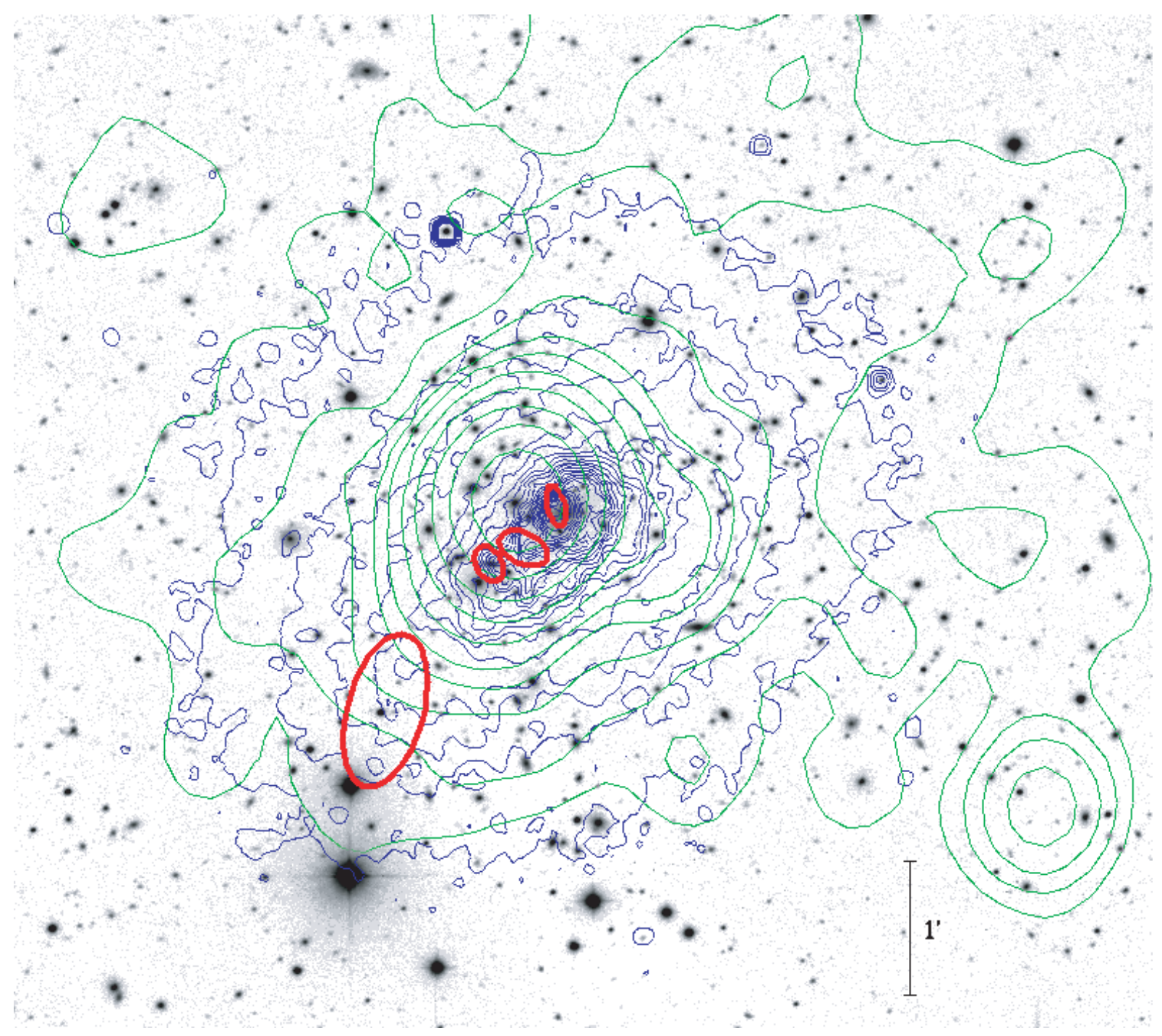

Fig. 10. $R$-band image of the cluster A2219 with, superimposed, the contour levels of the Chandra image (blue) and of a low-resolution VLA image (green) by Bacchi et al. (2003). The red ellipses represent the structures detected by Wavdetect. North is at top and East to left.

we verify that the two subsamples do not differ in the distribution of galaxy positions by using the 2DKS-test. Figure 14 shows the distribution of spectroscopically-classified galaxies: $4 / 25$ and $7 / 21$ are classified as active in the central and external cluster regions (within and outside $2^{\prime}$ ). Three central active galaxies are located in the NW filament of high velocity galaxies in the central region, corresponding to the NW subclump identified in the two-dimensional analysis of Sect. 3.3. The fourth galaxy (No. 81) lies at the SE of the cD galaxy.

For the interpretation of spectra, the only detected $e$-galaxy is very blue (the bluest within this sample) as generally found for starburst models. Nine of ten $a$-galaxies are classified as blue according to our definition (see Fig. 7). Thus their spectra can be explained by "post-starburst" models which reproduce strong $\mathrm{H} \delta E W$ and no significant emission lines in a quiescent phase soon after a starburst (about between a few Myr and 1.5 Gyr; e.g. Poggianti et al. 1999). Alternatively, strong $\mathrm{H} \delta E W$ could be obtained during a period of 1-2 Gyr which follows the end of star formation after a $1 \mathrm{Gyr}$ period of constant star formation (Morris et al. 1998).
Galaxy No. 81 shows the strongest $\mathrm{H} \delta E W$, and it is therefore spectroscopically classified as " $a$ ". However, it also shows evidence of mild [OII] emission, $E W \simeq 3 \AA$. It would have been classified " $e(a)$ " in the scheme of Poggianti et al. (1999). The galaxy coincides with one of the X-ray peaks, identified at the smallest wavelet scale. The X-ray emission could therefore be point-like, and consistent with emission from an AGN. The lack of strong emission lines could be explained if there is substantial dust obscuration. This interpretation is consistent with the fact that $e(a)$ galaxies are generally associated with dusty star-forming galaxies (Poggianti et al. 1999). The AGN emission should then be re-emitted in the infrared (IR). Although galaxy No. 81 was not detected at $15 \mu \mathrm{m}$ with ISOCAM by Barvainis et al. (1999), one can see low-level isocontours of mid-IR emission centered on this galaxy in Fig. 4 of Barvainis et al. (1999). Given that their rms noise level is $\approx 0.11 \mathrm{mJy}$, and their faintest detected source has a $15 \mu \mathrm{m}$ flux density of $0.53 \mathrm{mJy}$, the $15 \mu \mathrm{m}$ flux density of galaxy 81 should be $\lesssim 0.4$ $0.5 \mathrm{mJy}$. 


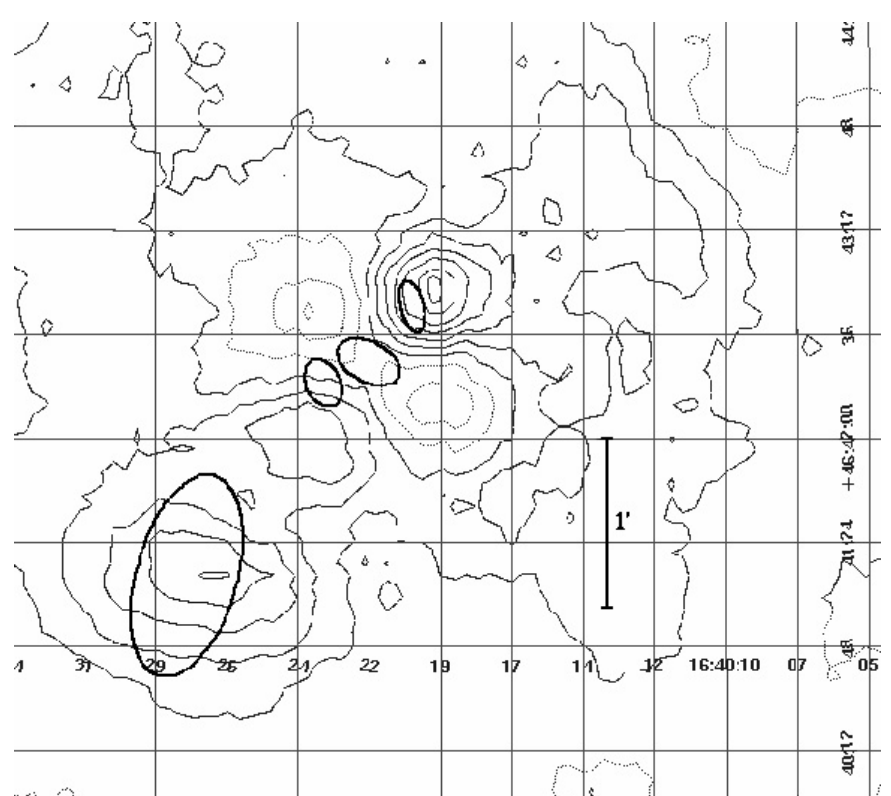

Fig. 11. Residual map of the elliptical Beta model fit (see text). Solid and dotted contour lines indicate regions with positive and negative residuals, respectively. Ellipses represent the structures detected by Wavdetect.

Of the 5 mid-IR sources detected by Barvainis et al. (1999) in the field of A2219, three are in our spectroscopic sample. These are the galaxies No. 42, 45 and 72 (respectively No. 1, 2 , and 3 in Barvainis et al. Table 1). Only galaxy No. 42 is a cluster member. The other two are foreground galaxies. Galaxy No. 42 is an $a$-type galaxy, with an $\mathrm{H} \delta E W \simeq 8 \AA$. Its spectrum shows evidence of a mild [OII] emission, $E W \simeq 3 \AA$. It would have been classified " $e(a)$ " in the scheme of Poggianti et al. (1999). The flux in the [OII] line can be used to compute the star formation rate of the galaxy, using the relation of Kennicutt (1998). We find $S F R \simeq 2 \pm 1 h^{-2} M_{\odot} \mathrm{yr}^{-1}$.

We have examined the optical-mid-IR spectral energy distribution (SED) of galaxy No. 42 comparing the observed data (taken from Barvainis et al. 1999) with the GRASIL models of Silva et al. (1998). The GRASIL model takes into account the effects of several kinds of dust particles on the reprocessing and obscuration of the stellar radiation (see: http://web.pd.astro.it/granato/grasil/ grasil.html). We find that the observed SED of galaxy No. 42 is best-fitted by a model of a young (3 Gyr-old) early-type spiral, with significant ongoing star formation (see Fig. 15). The best-fit model SED is used to compute the galaxy total IR luminosity, $\simeq 4 \times 10^{10} h^{-2} L_{\odot}$. The galaxy star formation rate, $S F R$, is derived from the total IR luminosity using the relation of Kennicutt (1998). We obtain $S F R \simeq 7.5 \pm$ $0.9 h^{-2} M_{\odot} \mathrm{yr}^{-1}$. This value is about 4 times higher than the estimate we obtain from the [OII] line flux. This is expected because of significant dust extinction at short wavelengths. From the best-fit model SED we estimate that dust extinction reduces the flux in the [OII] line by a factor $\sim 3$.

We conclude that galaxy No. 42 is a dusty star-forming galaxy, rather than a post-starburst galaxy. We thus find that both $e(a)$ galaxies of our sample are dusty, active galaxies, a
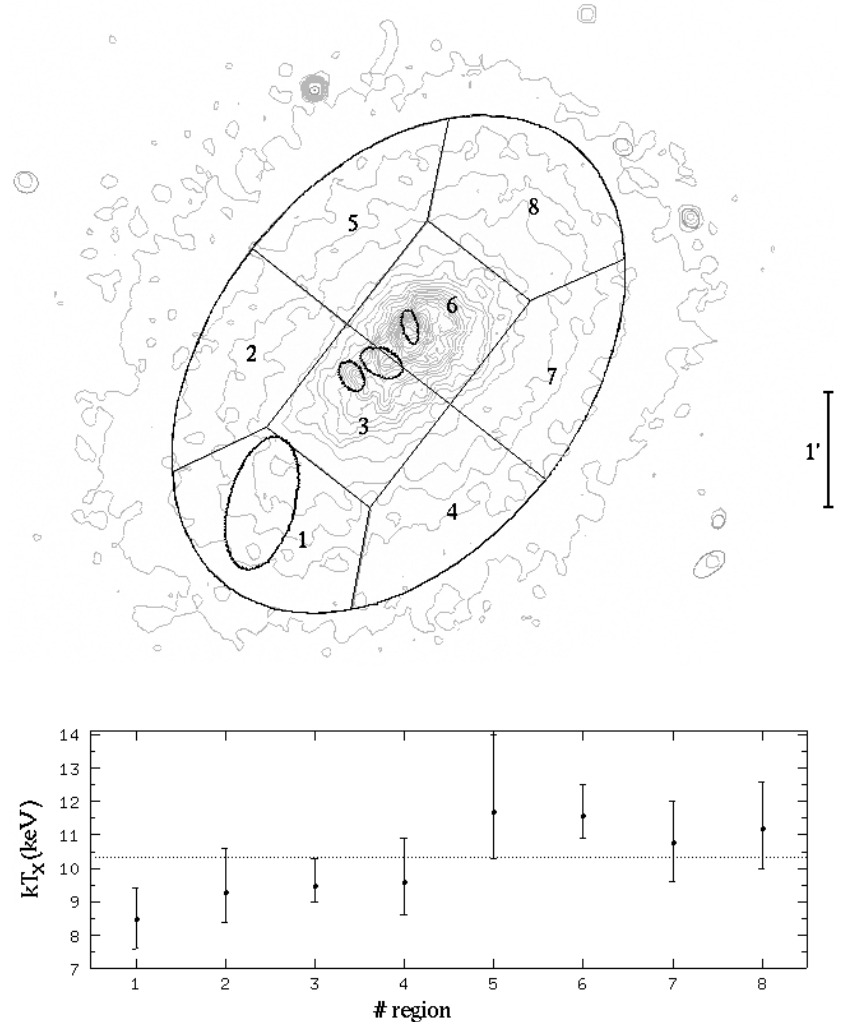

Fig. 12. Temperature map of A2219. The cluster area is divided in eight numbered sectors (top panel). Sectors are superimposed to the contour levels of the Chandra image. Ellipses show the four structures detected in the X-ray image. The graph in the bottom panel shows the temperature computed in each sector.

finding that supports the interpretation given by Poggianti et al. (1999) for these kind of galaxies.

With our spectral classification of a subset of A2219 galaxies, we can try to shed light on the nature of the "blue" galaxies which are responsible for the Butcher-Oemler (BO, hereafter) effect (Butcher \& Oemler 1978). We have translated the original Butcher \& Oemler definition of "blue" galaxies to the cluster redshift and the $B$ and $R$ filters, using the tables of Fukugita et al. (1995), and taking into account the Galactic absorption in the direction of A2219 (as given in the NASA Extragalactic Database). The dotted lines in Fig. 7 delimit the region of the colour-magnitude diagram where the $\mathrm{BO}$ galaxies are located. One can see that the BO population is of a mixed nature. First, there is a substantial number of field galaxies, of all spectroscopic types. Among BO cluster members, the dominant population is that of $a$-type galaxies. As we have shown, two of these $a$-type galaxies are in fact dusty active galaxies, rather than pure post-starburst objects. We thus conclude that the BO population of blue galaxies in the cluster A2219 is mostly composed of galaxies having experienced a recent starburst. In some of these galaxies the star-forming activity is not over yet, although partly hidden by dust.

For radio galaxies in the cluster, Bacchi et al. (2003) confirm the presence of three radio galaxies at the cluster center of A2219 (see Owen et al. 1992) identified in our catalogue with No. 51, 77, 85 (i.e. S3, S4, and S6 respectively in 


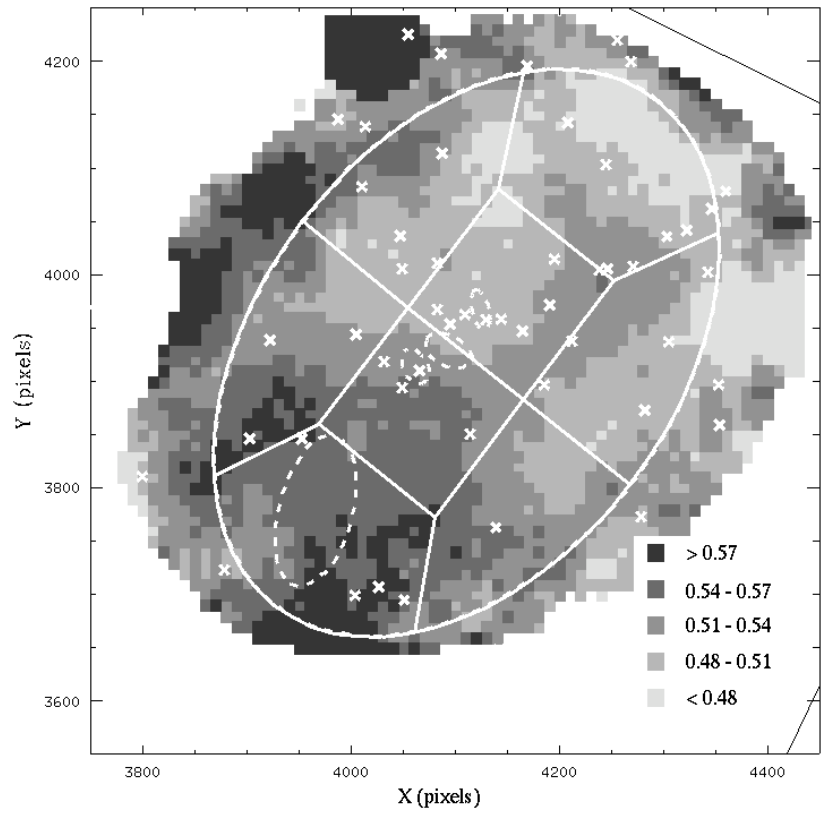

Fig. 13. Softness ratio map of A2219 (see text). Superimposed to the map are the eight sectors (see Fig. 12). Dashed ellipses are the structures detected in the X-ray image and crosses show the positions of the cluster members.

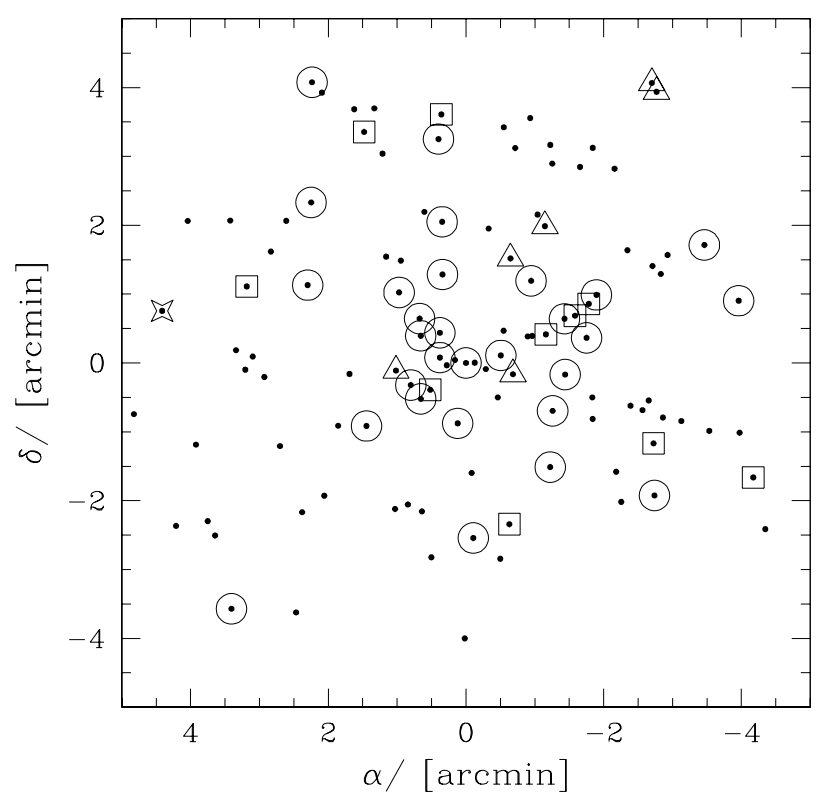

Fig. 14. Spatial distribution on the sky of 113 cluster members. Large symbols denotes spectroscopic classified galaxies: circles, triangles, squares, and stars indicate $k-, i_{-}, a-$, and $e$-galaxies, respectively.

Fig. 9 by Bacchi et al. 2003). The northernmost galaxy No. 77, which lies in the low-velocity tail of the cluster velocity distribution $\left(v=62364 \mathrm{~km} \mathrm{~s}^{-1}\right)$, shows a tailed structure pointing towards the NW, with a sharp bend towards the NE (see Owen \& Ledlow 1997). The galaxy No. 85 is the second brightest galaxy of the cluster $\left(\sim 17 \mathrm{R}\right.$ mag) and lies at $<1^{\prime}$ SE separation from the cD galaxy.

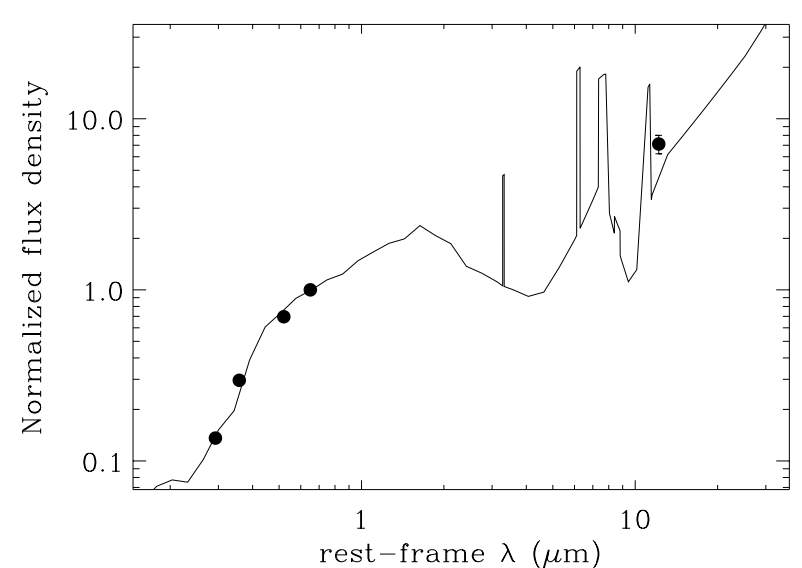

Fig. 15. Optical-mid IR spectral energy distribution of galaxy No. 42 . The best-fit GRASIL model is also shown (solid tree).

\section{Discussion and conclusion}

We analyze the internal dynamics of A2219 on the basis of spectroscopic data for 132 galaxies in a cluster region of $\sim 5^{\prime}\left(\sim 0.8 h^{-1} \mathrm{Mpc}\right.$ at the cluster distance) centered on the $\mathrm{cD}$ galaxy. Out of 132 spectra, 27 galaxies come from new observations carried out at the TNG. We find that A2219 appears as a peak in the velocity space at $z=0.225$, and select 113 cluster members. The investigation of the dynamical status is also performed using X-ray data stored in the Chandra archive. Further valuable information comes from other bands - optical photometric, infrared, and radio data - which are analyzed and/or discussed, too.

The value we obtain for the line-of-sight velocity dispersion $\sigma_{v} \sim 1400 \mathrm{~km} \mathrm{~s}^{-1}$ is in the high-tail of the distribution of cluster velocity dispersions (see Fadda et al. 1996; Mazure et al. 1996; Girardi \& Mezzetti 2001). This global value of $\sigma_{v}$ is consistent with the average value of $T_{\mathrm{X}} \sim 10 \mathrm{keV}$ coming from the X-ray analysis $\left(\beta_{\text {spec }}=1.10_{-0.12}^{+0.15}\right.$, see also Fig. 4) and with the value of $L_{X}(0.1-2.4 \mathrm{keV}) \sim 5 \times 10^{44} h^{-2} \mathrm{erg} \mathrm{s}^{-1}$ (Ebeling et al. 1996), converted to $L_{\mathrm{X}}$, bol $\sim 2 \times 10^{45} h^{-2} \mathrm{erg} \mathrm{s}^{-1}$ (see $L_{\mathrm{X}^{-}}$ $\sigma_{v}$ relation by, e.g., Wu et al. 1999; Girardi \& Mezzetti 2001).

Therefore, on the basis of global properties only, we would conclude that A2219 is not far from the dynamical equilibrium and would trust the large virial mass estimate $M\left(\sim 2.2 h^{-1} \mathrm{Mpc}\right) \sim 2.8 \times 10^{15} h^{-1} M_{\odot}$. However, the high values of $\sigma_{v}, T_{\mathrm{X}}$, and $L_{\mathrm{X}}$ could be due to the expected increase during a cluster-merging phase (e.g. Schindler \& Böhringer 1993; Schindler \& Müller 1993). Their agreement could be a coincidence, and our virial mass estimate an overestimate of the true value.

We find observational evidence that the cluster is not dynamically relaxed. Our optical and X-ray analyses show that A2219 is elongated in the SE-NW direction. This elongation is seen in the spatial distribution of the colour-selected likely cluster members, the shape of the cD galaxy, the X-ray contour levels of the Chandra image, the gradient in the velocity dispersion and in the X-ray temperature. In particular the ellipticity of X-ray isophotes is significantly higher than 0 and corresponds to an axis-ratio $=0.66$, which is much lower than the usual value for galaxy clusters (median axis-ratio with $99 \%$ c.l. 
errors $=0.82_{-0.02}^{+0.04}$ from Mohr et al. 1995). Note that Dahle et al. (2002) found that the mass distribution of this cluster recovered from a weak lensing analysis appears even more elongated than the light and galaxy number density distribution.

Very interestingly, the optical data show a somewhat smaller PA than X-ray data $\left(\sim 110^{\circ}\right.$ and $\sim 130^{\circ}$, respectively). A different direction in the elongation of light and hot gas distribution could be the result of a cluster merger in an advanced phase as shown by numerical simulations where collisional and collisionless components react in different way (e.g. Ricker \& Sarazin 2001; Schindler 2002). In fact, the evidence of an ongoing merger in the cluster core was first suggested by Smail et al. (1995) to explain the difference they find in the orientation of the mass and gas distributions ( $\mathrm{PA}=115$ and 128 degrees), as obtained from gravitational lensing analysis and ROSAT/HRI $\mathrm{X}$-ray data, respectively.

For the presence of the velocity gradient roughly in the E$\mathrm{W}$ direction, it suggests that the structures are moving into the plane of the sky at $\sim 45^{\circ}$ (e.g. Quintana et al. 1996; Roettiger $\&$ Flores 2000). Intermediate angles are the most suitable to allow detection of the cluster elongation and the velocity gradient during a merging phase.

The hypothesis of an advanced-phase merging is also supported by the lack of a cool center in the cluster (see also Allen \& Fabian 1998), as expected since strong cluster mergers disrupt the cool cores (Peres et al. 1998; Roettiger et al. 1996), and the existence in the core of several star-forming or poststarburst galaxies, whose activity could be produced by the cluster merger (e.g. Bekki 1999). In particular, three of these active galaxies are part of the filament $\mathrm{NW}$ of the $\mathrm{cD}$, also visible as a cool component in the X-ray softness ratio map (see Figs. 8 and 13), suggesting that this structure might be already involved in a merger event. Moreover, the shape of the radio tail of the radio galaxy No. 77 indicates that the velocity of this galaxy was originally pointing towards the cluster center (cD galaxy), but had suddenly changed direction, probably because of the cluster merger.

The structure we find in the cluster core could be related to the cluster merger, too. Considering optical data, the DresslerSchectman test shows the possible existence of a substructure located at SE, very close to the $\mathrm{cD}$ galaxy. For X-ray data, our wavelet multi-scale analysis reveals three significant emission peaks located within $1^{\prime}$ from the cluster center. Of these, two peaks are possibly connected to intrinsic X-ray emission from the $\mathrm{cD}$ and the active galaxy No. 81, but no optical counterpart is found for the third, intermediate peak. Our wavelet $\mathrm{X}$-ray analysis also detects an external diffuse substructure located $2^{\prime} \mathrm{SE}$ of the $\mathrm{cD}$ galaxy. We suggest that this structure is a premerging clump. It coincides with the cold SE X-ray region and thus could be well explained by the two-dimensional superposition of a cold clump in the foreground of the main cluster. The presence of this foreground clump could also explain why, on average, the velocity to the East is lower than the West since at least for some galaxies the low velocity is of cosmological rather than of kinematic origin. These anomalous low velocity galaxies could also produce a spurious enhancement of the observed velocity dispersion in the SE region (see Table 2). A population of very close foreground galaxies could also explain why very red galaxies strangely differ from notvery red galaxies both in kinematic and spatial properties (see Sect. 3.3). While the former are really associated with the main structure of A2219, the latter could be mixed with the clump members, i.e. a population of foreground - and thus less red - galaxies. Unfortunately, we have very poor redshift information in the SE region to confirm our hypothesis and identify clump members.

On a much larger scale, the cluster is not so well isolated in the redshift space, and the next possible merging clump might be the one at $z \sim 0.19$ (see Fig. 2).

Summarizing the above evidence, we suggest a complex merging scenario for A2219 involving many clumps, possibly in different dynamical states. We suggest that the main, original structure (hosting the cD galaxy) is subject to an ongoing merger with a few clumps aligned in a foreground filament obliquely oriented with respect to the line-of-sight; the filament projected on the sky lies in the SE-NW direction. In this scenario, the merging with one or more clumps is already in a very advanced phase, well after the first core passage, while one or more clumps are still in a pre-merging phase.

In the context of the above scenario, we argue that the presence of a radio-halo in A2219 is related to the existence of an ongoing, very advanced phase of merging between the main cluster and one or more groups. In fact, the elongation of radio map contours (see Fig. 10) is in the same direction as the X-ray surface brightness contours. Moreover, the time necessary to re-accelerate the electrons producing the radio-halo as a consequence of the cluster merger ( $\sim 1 \mathrm{Gyr})$ is comparable to that needed to obtain post-starburst spectral signatures in galaxies of which A2219 is rich (see Sect. 5).

In conclusion, our scenario supports the view of the connection between extended radio emission and merging phenomena in galaxy clusters.

Acknowledgements. We thank Luca di Fabrizio for $B$ and $R$ calibration observations at the TNG telescope and the anonymous referee for useful suggestions and comments. We particularly thank Ian Smail for providing us accurate Palomar 5-m telescope photometry. Work partially supported by the Italian Ministry of Education, University, and Research (MIUR, grant COFIN2001028932 "Clusters and groups of galaxies, the interplay of dark and baryonic matter"), by the Italian Space Agency (ASI), and by INAF (Istituto Nazionale di Astrofisica) through grant D4/03/IS. This publication makes use of data accessed as Guest User at the Canadian Astronomy Data Center, which is operated by the Dominion Astrophysical Observatory for the National Research Council of Canada's Herzberg Institute of Astrophysics (http://cadcwww.dao.nrc.ca/cfht/cfht.html), as well as of data obtained from the Chandra data archive at the NASA Chandra X-ray center (http: //asc . harvard. edu/cda/).

\section{References}

Abell, G. O., Corwin, H. G. Jr., \& Olowin, R. P. 1989, ApJS, 70, 1

Allen, S. W., Edge, A. C., Fabian, A. C., et al. 1992, MNRAS, 259, 67

Allen, S. W., \& Fabian, A. C. 1998, MNRAS, 297, L57

Allen, S. W. 2000, MNRAS, 315, 269 
Bacchi, M., Feretti, L., Giovannini, G., \& Govoni, F. 2003, A\&A, 400, 465

Balogh, M. L., Morris, S. L., Yee, H. K. C., Carlberg, R. G., \& Ellingson, E. 1999, ApJ, 527, 54

Bardelli, S., Zucca, E., Vettolani, G., et al. 1994, MNRAS, 267, 665

Barrena, R., Biviano, A., Ramella, M., Falco, E. E., \& Seitz, S. 2002, A\&A, 386, 816

Barvainis, R., Antonucci, R., \& Helou, G. 1999, AJ, 118, 645

Beers, T. C., Flynn, K., \& Gebhardt, K. 1990, AJ, 100, 32

Beers, T. C., Forman, W., Huchra, J. P., Jones, C., \& Gebhardt, K. 1991, AJ, 102, 1581

Bekki, K. 1999, ApJ, 510, L15

Bertin, E., \& Arnouts, S. 1996, A\&AS, 117, 393

Böhringer, H., \& Schuecker, P. 2002, in Merging Processes in Galaxy Clusters, ed. L. Feretti, I. M. Gioia, \& G. Giovannini (The Netherlands: Kluwer Ac. Pub.), 133

Buote, D. A. 2002, in Merging Processes in Galaxy Clusters, ed. L. Feretti, I. M. Gioia, \& G. Giovannini (The Netherlands: Kluwer Ac. Pub.), 79

Butcher, H., \& Oemler, A. Jr. 1978, ApJ, 219, 18

Carlberg, R. G., Yee, H. K. C., Ellingson, E., et al. 1997, ApJ, 476, L7

Colless, M., \& Dunn, A. M. 1996, ApJ, 458, 435

Couch, W. J., Ellis, R. S., Sharples, R. M., \& Smail, I. 1994, ApJ, 430, 121

Dahle, H., Kaiser, N., Irgens, R. J., Lilje, P. B., \& Maddox, S. J. 2002, ApJS, 139, 313

Danese, L., De Zotti, C., \& di Tullio, G. 1980, A\&A, 82, 322

den Hartog, R., \& Katgert, P. 1996, MNRAS, 279, 349

Dickey, J. M., \& Lockman, F. J. 1990, ARA\&A, 28, 215

Dressler, A., \& Shectman, S. A. 1988, AJ, 95, 985

Dressler, A., Smail, I., Poggianti, B. M., et al. 1999, ApJS, 122, 51

Ebeling, H., Voges, W., Böhringer, H., et al. 1996, MNRAS, 281, 799

Ellingson, E., \& Yee, H. K. C. 1994, ApJS, 92, 33

Ensslin, T. A. 2000, in Cluster Mergers and their Connection to Radio Sources, 24th meeting of the IAU, Joint Discussion 10, August 2000, Manchester, England

Ensslin, T. A., \& Röttgering, H. 2002, A\&A, 396, 83

Evrard, A. E., \& Gioia, I. M. 2002, in Merging Processes in Galaxy Clusters, ed. L. Feretti, I. M. Gioia, \& G. Giovannini (The Netherlands: Kluwer Ac. Pub.), Clusters, Cosmology and Mergers

Fadda, D., Girardi, M., Giuricin, G., Mardirossian, F., \& Mezzetti, M. 1996, ApJ, 473, 670

Fasano, G., \& Franceschini, A. 1987, MNRAS, 225, 155

Feretti, L., \& Giovannini, G. 1996, Extragalactic radio sources, proceedings of the 175th Symposium of the International Astronomical Union, held in Bologna, Italy, 10-14 October 1995, ed. R. D. Ekers, C. Fanti, \& L. Padrielli (Kluwer Academic Publishers), 333

Feretti, L. 1999, MPE Rep., No. 271

Feretti, L. 2002, The Universe at Low Radio Frequencies, Proceedings of IAU Symp., 199, held 30 Nov.-4 Dec. 1999, Pune, India, ed. A. Pramesh Rao, G. Swarup, \& Gopal-Krishna, 133

Fukugita, M., Shimasaku, K., \& Ichikawa, T. 1995, PASP, 107, 945

Gebhardt, K., \& Beers, T. C. 1991, ApJ, 383, 72

Giovannini, G., Tordi, M., \& Feretti, L. 1999, New Astronomy, 4, 141

Giovannini, G., \& Feretti, L. 2002, in Merging Processes in Galaxy Clusters, ed. L. Feretti, I. M. Gioia, \& G. Giovannini (The Netherlands: Kluwer Ac. Pub.), 197

Girardi, M., Fadda, D., Giuricin, G., et al. 1996, ApJ, 457, 61

Girardi, M., Giuricin, G., Mardirossian, F., Mezzetti, M., \& Boschin, W. 1998, ApJ, 505, 74
Girardi, M., \& Mezzetti, M. 2001, ApJ, 548, 79

Girardi, M., \& Biviano, A. 2002, in Merging Processes in Galaxy Clusters, ed. L. Feretti, I. M. Gioia, \& G. Giovannini (The Netherlands: Kluwer Ac. Pub.), 39

Gómez, P. L., Hughes, J. P., \& Birkinshaw, M. 2000, ApJ, 540, 726

Govoni, F., Feretti, L., Giovannini, G., et al. 2001, A\&A, 376, 803

Hammer, F., Flores, H., Lilly, S. J., et al. 1997, ApJ, 481, 49

Kennicutt, R. C. 1992, ApJS, 79, 225

Kennicutt, R. C. 1998, ARA\&A, 36, 189

Malamuth, E. M., Kriss, G. A., Dixon, W., Van Dyke, Ferguson, H. C., \& Ritchie, C. 1992, AJ, 104, 495

Mazure, A., Katgert, P., den Hartog, P., et al. 1996, A\&A, 310, 31

Mercurio, A., Girardi, M., Boschin, W., Merluzzi, P., \& Busarello, G. 2003, A\&A, 397, 431

Mohr, J. J., Evrard, A. E., Fabricant, D. G., \& Geller, M. J. 1995, ApJ, 447,7

Morris, S. L., Hutchings, J. B., Carlberg, R. G., et al. 1998, ApJ, 507, 84

Owen, F. N., White, R. A., \& Burns, J. O. 1992, ApJS, 80, 501

Owen, F. N., \& Ledlow, M. J. 1997, ApJS, 108, 41

Peres, C. B., Fabian, A. C., Edge, A. C., et al. 1998, MNRAS, 298, 416

Pisani, A. 1993, MNRAS, 265, 706

Pisani, A. 1996, MNRAS, 278, 697

Poggianti, B. M., Smail, I., Dressler, A., et al. 1999, ApJ, 518, 576

Postman, M., Lubin, L. M., \& Oke, J. B. 1998, AJ, 116, 560

Press, W. H., Teukolsky, S. A., Vetterling, W. T., \& Flannery, B. P. 1992, in Numerical Recipes (Second edition) (Cambridge University Press)

Quintana, H., Carrasco, E. R., \& Reisenegger, A. 2000, AJ, 120, 511

Quintana, H., Ramirez, A., \& Way, M. J. 1996, AJ, 112, 36

Raymond, J. C., \& Smith, B. W. 1977, ApJS, 35, 419

Ricker, P. M., \& Sarazin, C. L. 2001, ApJ, 561, 621

Rizza, E., Burns, J. O., Ledlow, M. J., et al. 1998, MNRAS, 301, 328

Roettiger, K., Burns, J. O., \& Loken, C. 1996, ApJ, 473, 651

Roettiger, K., Loken, C., \& Burns, J. O. 1997, ApJS, 109, 307

Roettiger, K., \& Flores, R. 2000, ApJ, 538, 92

Sarazin, C. L. 2002, in Merging Processes in Galaxy Clusters, ed. L. Feretti, I. M. Gioia, \& G. Giovannini (The Netherlands: Kluwer Ac. Pub.), 1

Schindler, S., \& Böhringer, H. 1993, A\&A, 269, 83

Schindler, S., \& Müller, E. 1993, A\&A, 272, 137

Schindler, S. 2002, in Merging Processes in Galaxy Clusters, ed. L. Feretti, I. M. Gioia, \& G. Giovannini (The Netherlands: Kluwer Ac. Pub.), 229

Schuecker, P., Böhringer, H., Reiprich, T. H., \& Feretti, L. 2001, A\&A, 378, 408

Silva, L., Granato, G. L., Bressan, A., \& Danese, L. 1998, ApJ, 509, 103

Smail, I., Hogg, D. W., Blandford, R., et al. 1995, MNRAS, 277, 1

Smail, I., Edge, A. C., Ellis, R. S., \& Blandford, R. D. 1998, MNRAS, 293, 124

Terlevich, A. I., Kuntschner, H., Bower, R. G., Caldwell, N., \& Sharples, R. M. 1999, MNRAS, 310, 445

Tonry, J., \& Davis, M. 1979, ApJ, 1511

White, D. A. 2000, MNRAS, 312, 663

White, S. D. M. 1997, in The Evolution of the Universe, report of the Dahlem Workshop on the Evolution of the Universe, Berlin, September 10-15, 1995, ed. G. Borner, \& S. Gottlober (New York: J. Wiley), 227

Wu, X.-P., Xue, Y.-J., \& Fang, L.-Z. 1999, ApJ, 524, 22 